\title{
Femtosecond tuning of Cr:colquiriite lasers with AlGaAs-based saturable Bragg reflectors
}

\author{
Umit Demirbas, ${ }^{1}$ Gale S. Petrich, ${ }^{1}$ Duo Li, ${ }^{1}$ Alphan Sennaroglu, ${ }^{1,2}$ Leslie A. Kolodziejski, ${ }^{1}$ \\ Franz X. Kärtner, ${ }^{1}$ and James G. Fujimoto ${ }^{1, *}$ \\ ${ }^{1}$ Department of Electrical Engineering and Computer Science and Research Laboratory of Electronics, \\ Massachusetts Institute of Technology, Cambridge, Massachusetts 02139, USA \\ ${ }^{2}$ Visiting from the Koç University, Istanbul 34450, Turkey \\ *Corresponding author: jgfuji@mit.edu
}

Received November 1, 2010; revised February 16, 2011; accepted February 17, 2011; posted February 17, 2011 (Doc. ID 137546); published April 4, 2011

\begin{abstract}
We present a detailed experimental study of femtosecond tuning with Cr:LiSAF and Cr:LiCAF gain media using $\mathrm{Al}_{0.95} \mathrm{Ga}_{0.05} \mathrm{As} / \mathrm{Al}_{0.17} \mathrm{Ga}_{0.83}$ As-based saturable Bragg reflectors. In the experiments with Cr:LiSAF gain media, femtosecond tuning ranges of $803-831 \mathrm{~nm}(28 \mathrm{~nm}), 828-873 \mathrm{~nm}(45 \mathrm{~nm})$ and $890-923 \mathrm{~nm}(33 \mathrm{~nm})$ were demonstrated using three different saturable Bragg reflectors with reflectivity bands centered at around 800, 850, and $910 \mathrm{~nm}$, respectively. With Cr:LiCAF gain medium, a femtosecond tuning range of $767-817 \mathrm{~nm}(50 \mathrm{~nm})$ was demonstrated using the $800 \mathrm{~nm}$ saturable Bragg reflector. Pulses as short as 26 fs with Cr:LiSAF and $39 \mathrm{fs}$ with Cr:LiCAF have been obtained, which we believe are the shortest pulses reported from Cr:colquiriite laser systems mode locked with saturable

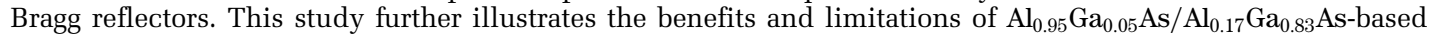
saturable Bragg reflectors for use in Cr:colquiriites. (c) 2011 Optical Society of America
\end{abstract}

OCIS codes: $\quad 140.3460,140.5680,140.3580,140.3600,140.3480,140.4050$.

\section{INTRODUCTION}

Commercial mode-locked Ti:sapphire laser oscillators can provide $\sim 100 \mathrm{fs}$ long pulses, with hundreds of kilowatts of peak power and broad tunability from 680 to $1080 \mathrm{~nm}$. Broad tunability in the mode-locked regime is quite important for applications such as multiphoton microscopy [1] $]$, time-resolved photoluminescence, or pump-probe spectroscopy. However, the current cost of Ti:sapphire technology is a barrier to its widespread use, limiting progress in many areas of science and technology.

$\mathrm{Cr}^{3+}:$ colquiriite $\left(\mathrm{Cr}^{3+}: \mathrm{LiSAF}\right.$ [2], $\mathrm{Cr}^{3+}: \mathrm{LiSGaF}$ [3], and $\mathrm{Cr}^{3+}$ : LiCAF [4]) lasers are a promising low-cost alternative to Ti:sapphire due to (i) their broad emission bands covering the $750-1050 \mathrm{~nm}$ spectral region and (ii) the possibility of direct diode pumping with low-cost laser diodes emitting around $650 \mathrm{~nm}$ [5-20]. Besides enabling a significant reduction in cost, direct pumping with high-brightness diodes also greatly improves electrical-to-optical conversion efficiency. Among the Cr:colquiriites, Cr:LiSAF has the broadest gain and its demonstrated continuous-wave $(\mathrm{cw})$ tuning range extends from 775 to $1042 \mathrm{~nm}$ [18] (extending to $1065 \mathrm{~nm}$ in pulsed operation [21]). Cr:LiC $\overline{\mathrm{AF}}$ has received attention due to its blueshifted emission spectrum, which enables a cw tuning range of 754-871 nm [18] (extending to $720 \mathrm{~nm}$ in quasi-cw operation [4]). To date, most femtosecond laser development efforts with Cr:colquiriites have focused on fixed wavelength operation near the gain peak, which is around $800 \mathrm{~nm}$ for Cr:LiCAF and $850 \mathrm{~nm}$ for Cr:LiSAF and Cr:LiSGaF.

With Kerr-lens mode-locked (KLM) Cr:colquiriite lasers, pulses as short as $\sim 10 \mathrm{fs}$ [22-24] and femtosecond tuning ranges of 835-910 [25] and 809-910 nm [26] have been demonstrated. As an example, in the study by Robertson et al., $\sim 1 \mathrm{~W}$ of near diffraction-limited $\left(M^{2} \sim 2\right)$ pump power from two ta- pered laser diodes was used to pump a Cr:LiSAF laser [26]. The Cr:LiSAF laser cavity contained a prism pair for dispersion adjustment and a slit to tune the wavelength [26]. Within the tuning range (809-910 nm), the pulse widths varied between 70 and $170 \mathrm{fs}$ and pulse energies were in the $0.2-1.2 \mathrm{~nJ}$ range [26]. Since the Kerr-lensing mechanism does not impose a bandwidth limitation, the shortest pulses and the broadest tuning range from Cr:colquiriite lasers have been obtained from KLM systems. However, the main difficulty with KLM mode-locked Cr:colquiriite lasers is the long-term stability and robustness of the mode-locked laser system, due to the relatively low nonlinear refractive index $\left(n_{2}\right)$ of Cr:LiSGaF $\left(1.2 \times 10^{-16} \mathrm{~cm}^{2} / \mathrm{W}\right), \quad$ Cr:LiSAF $\quad\left(0.8 \times 10^{-16} \mathrm{~cm}^{2} / \mathrm{W}\right), \quad$ and Cr:LiCAF $\left(0.4 \times 10^{-16} \mathrm{~cm}^{2} / \mathrm{W}\right.$, which is 8 times smaller than Ti:sapphire [18,27]). Moreover, the efficiency (average output power/pump power) of the KLM Cr:LiSAF lasers is also relatively low due to the need for critical alignment of the cavity near its stability edge.

As an alternative to KLM, femtosecond pulses can be initiated using semiconductor saturable Bragg reflectors (SBRs) [28], which are also referred to as semiconductor saturable absorber mirrors (SESAMs) [29]. Advantages of using SBRs instead of KLM include self-starting, robust mode-locked operation with long-term stability, immunity to environmental fluctuations, reduced cavity alignment sensitivity, and higher efficiencies. Using low-cost, single-mode laser diodes that emit around $650 \mathrm{~nm}$ (optical power of $\sim 150 \mathrm{~mW}$ ), and SBRs for mode-locking, turn-key femtosecond Cr:colquiriite lasers, with sub-100-fs pulses and $20-40 \mathrm{~kW}$ peak power were obtained with electrical-to-optical conversion efficiencies above $8 \%[17,19]$. Once aligned, these lasers are quite stable and operate as a turn-key system for days to weeks, requiring little adjustment due to mechanical/thermal misalignments. To our knowledge, the only report of femtosecond tuning of SBR 
mode-locked Cr:colquiriite lasers is by Kopf et al., who demonstrated a tuning range from $\sim 825$ to $\sim 875 \mathrm{~nm}$ from an SBR mode-locked, multimode diode-pumped Cr:LiSAF laser [30]. However, this study used a prism pair and a slit for tuning, and for each central wavelength, the dispersion was optimized, making the cavity alignment sensitive and turn-key operation difficult.

This manuscript presents a detailed femtosecond tuning investigation of SBR mode-locked Cr:LiSAF and Cr:LiCAF lasers pumped by single-mode diodes. A quartz birefringent wave plate is used for wavelength tuning, which enables more robust operation. With the Cr:LiSAF gain medium, using an SBR centered at $850 \mathrm{~nm}$ (hereafter referred to as an $850 \mathrm{~nm}$ SBR), a femtosecond tuning range of $828-873 \mathrm{~nm}(45 \mathrm{~nm})$ was obtained with average pulse widths of $\sim 190 \mathrm{fs}$ and average pulse energies of $1.87 \mathrm{~nJ}$. By using an $800 \mathrm{~nm}$ SBR, the Cr:LiSAF laser could be tuned from 803 to $831 \mathrm{~nm}(28 \mathrm{~nm}$, average pulse width of $140 \mathrm{fs}$, average pulse energy of $1 \mathrm{~nJ}$ ). Furthermore, a $910 \mathrm{~nm}$ SBR resulted in a tuning range from 890 to $923 \mathrm{~nm}$ with the same laser. With the Cr:LiCAF gain medium, a modelocked tuning range of $767-817 \mathrm{~nm}(50 \mathrm{~nm})$, with average pulse widths of $\sim 133 \mathrm{fs}$ and average pulse energies of $1.48 \mathrm{~nJ}$ was obtained. To our knowledge, this study provides the first account of detailed femtosecond tuning experiments with SBR mode-locked Cr:colquiriite lasers. Furthermore, pulses as short as 26 and 39 fs were obtained with Cr:LiSAF and Cr:LiCAF lasers, respectively, which, to our knowledge, are the shortest pulses reported from SBR mode-locked Cr:colquiriite lasers.

This paper focuses on the femtosecond tuning results that were obtained with Cr:colquiriite lasers. We refer to [17-19, $\underline{31}]$ for a general description of SBR mode-locking of single-mode diode-pumped Cr:colquiriites and further discussion on $Q$-switching and multiple pulsing instabilities.

\section{FEMTOSECOND TUNING OF CR:LISAF NEAR 850 NM}

\section{A. Experimental Setup}

Figure 1 shows the schematic of the Cr:LiSAF laser used in the femtosecond tuning experiments near $850 \mathrm{~nm}$. The Cr:LiSAF crystal was pumped by four $640 \mathrm{~nm}$ (Hitachi HL6385DG) linearly polarized, single-mode diodes $\left(M^{2}<1.05\right)$ with circular outputs (D1-D4). The diodes have a rated output power of $150 \mathrm{~mW}$ with $280 \mathrm{~mA}$ of pump current at $25^{\circ} \mathrm{C}$. The laser diodes were cooled to $20^{\circ} \mathrm{C}$ by a thermoelectric cooler and operated at $350 \mathrm{~mA}$ to obtain $\sim 200 \mathrm{~mW}$ from each laser diode.
No degradation of the pump laser diodes was observed up to hundreds of hours of operation. The diode outputs were collimated with $4.5 \mathrm{~mm}$ focal length aspheric lenses and combined by polarizing beam splitter (PBS) cubes. Two $65 \mathrm{~mm}$ focal length lenses focused the pump beams into the Cr:LiSAF crystal. The $6 \mathrm{~mm}$ long Cr:LiSAF crystal had a Cr concentration of $1.5 \%$ and absorbed $>99 \%$ and $>77 \%(0.9 \times 85 \%)$ of the incident TM- and TE-polarized pump light at $640 \mathrm{~nm}$, respectively. An astigmatically compensated, x-folded laser cavity containing two curved pump mirrors (M1-M2, radius of curvature ROC $=75 \mathrm{~mm}$ ) was used. The cavity was first optimized for $\mathrm{cw}$ operation, generating $335 \mathrm{~mW}$ of output power with $800 \mathrm{~mW}$ of incident pump power $(\sim 720 \mathrm{~mW}$ of absorbed pump power) using a $3 \%$ output coupler. To initiate and sustain mode-locked operation, a $850 \mathrm{~nm}$ SBR was used. The SBR consisted of 25 pairs of $\mathrm{Al}_{0.95} \mathrm{Ga}_{0.05} \mathrm{As} / \mathrm{Al}_{0.17} \mathrm{Ga}_{0.83} \mathrm{As}$ quarterwave layers as a Bragg mirror stack and one layer of $25 \mathrm{~nm}$ thick GaAs as a saturable absorber. The epitaxial growth of the SBR was performed in a solid source, multiwafer, dualreactor molecular beam epitaxy (MBE) system (Veeco GEN 200), at typical AlGaAs growth temperatures. After the MBE growth, an additional $\mathrm{SiO}_{2}-\mathrm{TiO}_{2}$ pair was used as a highreflection (HR) coating on the surface. The calculated modulation depth of the $850 \mathrm{~nm}$ centered SBR was $0.8 \pm 0.2 \%$. For soliton pulse shaping, dispersion compensation was performed with custom-designed dispersion-compensating mirrors (DCMs) (M1-M5) with a group velocity dispersion (GVD) of $\sim-80 \mathrm{fs}^{2}$ per bounce $(800-940 \mathrm{~nm})[\underline{32}, \underline{33}]$. A $3 \mathrm{~mm}$ thick quartz birefringent plate, designed with the optic axis out of plane was used for tuning. The same $3 \%$ output coupler is also used in mode-locked tuning experiments.

\section{B. Results}

Using the $850 \mathrm{~nm}$ SBR, the central wavelength of the Cr:LiSAF laser could be tuned continuously from $\sim 828$ to $\sim 873 \mathrm{~nm}$ ( $\sim 45 \mathrm{~nm}$ tuning), by rotating the birefringent tuning plate. Figure 2 shows the laser pulse width and pulse energy over the tuning range. On average, the Cr:LiSAF laser generated $\sim 188$ fs pulses (assuming sech $^{2}$ pulse shape) with $\sim 170 \mathrm{~nm}$ of average power at a repetition rate of $91 \mathrm{MHz}(\sim 1.87 \mathrm{~nJ}$ pulse energy). Figure 3 shows the measured spectra over the tuning range along with the estimated total cavity GVD and the calculated small-signal SBR reflectivity. The total round-trip cavity dispersion was estimated to be $\sim-900 \mathrm{fs}^{2}$, where the estimate includes one bounce on the SBR, 18 bounces on the DCMs, $\sim 3 \mathrm{~m}$ of intracavity air, $12 \mathrm{~mm}$ of Cr:LiSAF material,

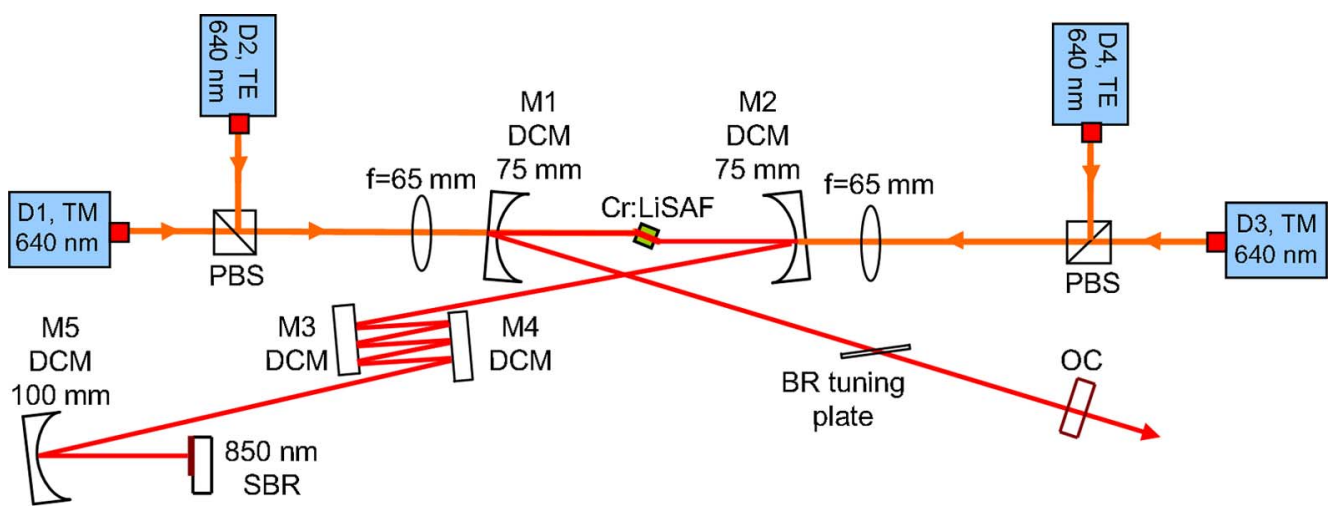

Fig. 1. (Color online) Schematic of the single-mode diode-pumped Cr:LiSAF laser used in femtosecond tuning experiments around $850 \mathrm{~nm}$. The estimated total cavity dispersion is $\sim-900 \mathrm{fs}^{2}$ per round trip. The total incident pump power on the crystal is $\sim 800 \mathrm{~mW}$. 


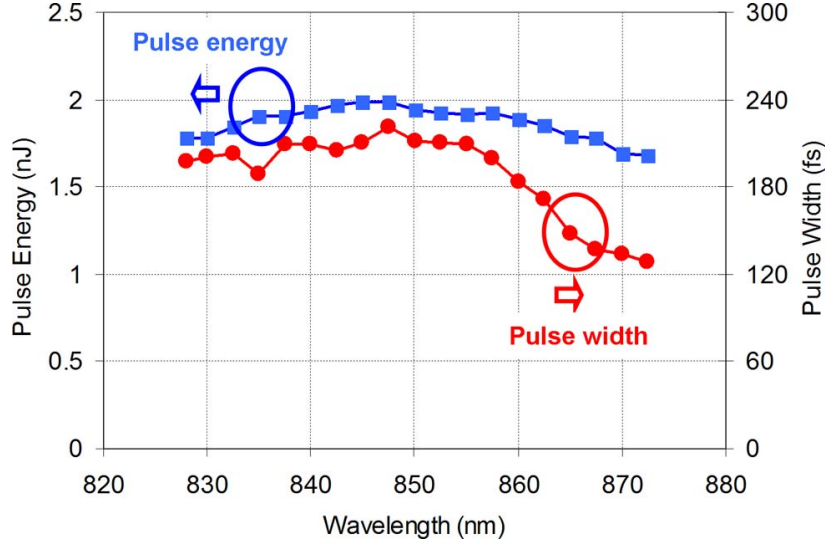

Fig. 2. (Color online) Femtosecond tuning results with the Cr:LiSAF laser using the $850 \mathrm{~nm}$ SBR. The total tuning range is $\sim 45 \mathrm{~nm}$ (828 to $873 \mathrm{~nm})$.

and $7.3 \mathrm{~mm}$ of quartz birefringent tuning plate. The total cavity GVD curve is relatively flat around $850 \mathrm{~nm}$ with a bandwidth of about $50 \mathrm{~nm}$, then varies significantly due to the relatively narrow dispersion bandwidth of the SBR. We believe that the tuning range was limited by the SBR reflectivity and the dispersion bandwidth on the short wavelength side. On the long wavelength side, when the laser was configured for operation above $873 \mathrm{~nm}$, the laser started to lase cw near $885 \mathrm{~nm}$. This is because the absorption edge of GaAs is around $870 \mathrm{~nm}$ and, hence, there is insufficient modulation in the SBR to enable mode-locking above $873 \mathrm{~nm}$. We anticipate that using absorber with an absorption band edge at a slightly longer wavelength (for example, an InGaAs absorber with a few percent indium, instead of pure GaAs), will increase the tuning range by $5-10 \mathrm{~nm}$ on the long wavelength side.

When the number of bounces on the DCM mirrors was decreased from 18 to 14 , the total estimated cavity GVD decreased from $\sim-900$ to $-600 \mathrm{fs}^{2}$. At this dispersion level, continuous tunability from 835 to $870 \mathrm{~nm}$ ( $~ 35 \mathrm{~nm}$ tuning) was obtained by rotating the tuning plate. The average pulse width decreased to $\sim 140$ femtoseconds and the average pulse energy was $\sim 1.8 \mathrm{~nJ}$ at a repetition rate of $\sim 110 \mathrm{MHz}$. Compared to the previous configuration, the tuning range was narrower, since more of the SBR bandwidth was required for the shorter pulses and mode-locking stability became more susceptible to dispersion changes. The Cr:LiSAF laser can be adjusted to

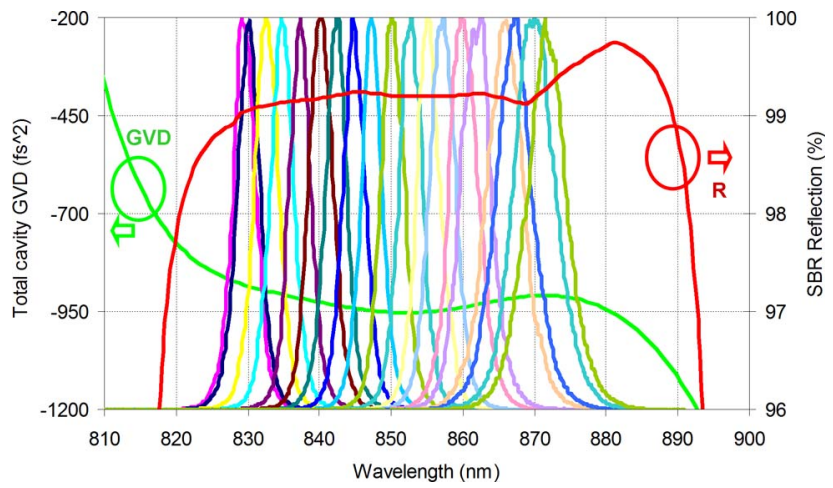

Fig. 3. (Color online) Typical spectra from the Cr:LiSAF laser, showing tunability of central wavelength from 828 to $873 \mathrm{~nm}$ with pulse durations of $<200 \mathrm{fs}$. The data were taken with the $850 \mathrm{~nm}$ SBR. The calculated small-signal reflectivity of the SBR and estimated total cavity GVD are also shown.
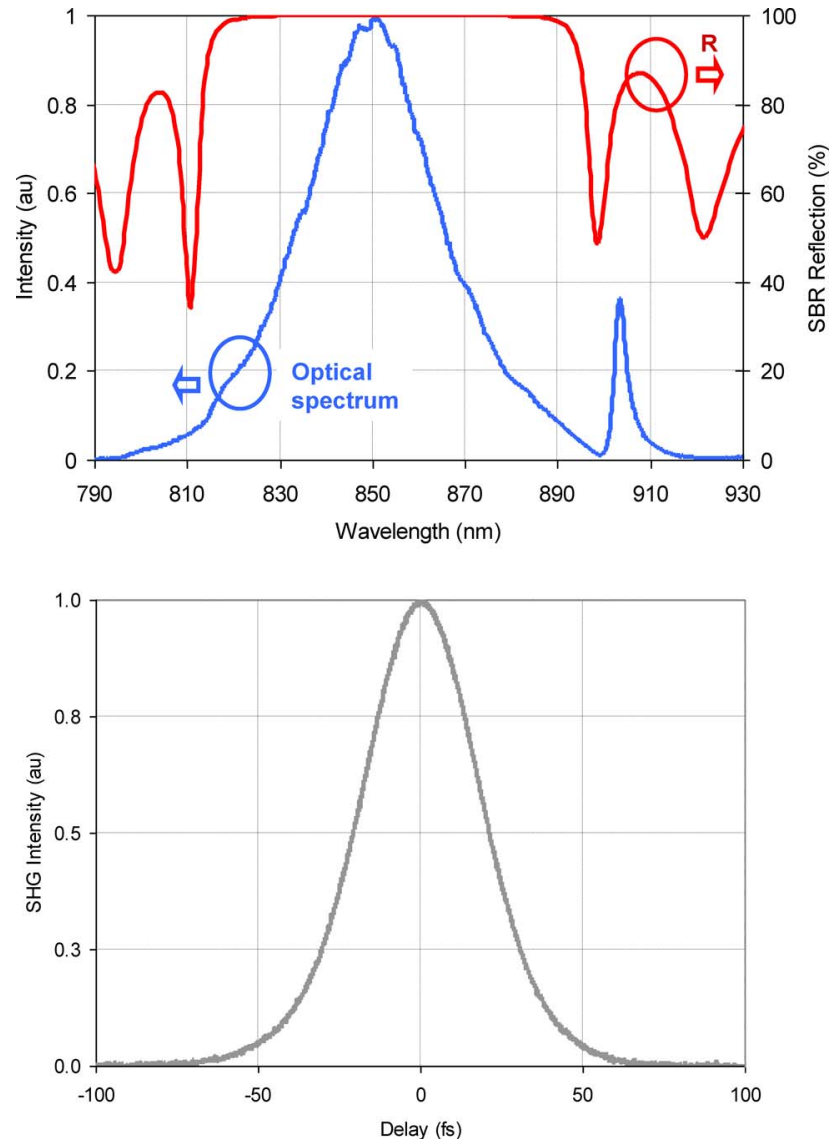

Fig. 4. (Color online) Top, optical spectrum for the $26 \mathrm{fs}, 1 \mathrm{~nJ}$ pulses obtained from the Cr:LiSAF laser with the $850 \mathrm{~nm}$ SBR. The calculated saturated reflectivity of the SBR is also shown. Bottom, measured background-free autocorrelation trace for the $26 \mathrm{fs}$ pulses.

emit even shorter pulses; however, the tuning range is narrower. For example, [18] demonstrates a $28 \mathrm{~nm}$ tuning of a Cr:LiSAF laser with sub-80-fs pulses using a similar SBR centered at $850 \mathrm{~nm}$ (Figs. 12 and 13 in [18]).

The Cr:LiSAF laser was also configured to produce sub30 -fs pulses with the $850 \mathrm{~nm}$ SBR. A fused-silica prism pair with $28 \mathrm{~cm}$ separation was used to adjust the intracavity dispersion (for a similar cavity design, see Fig. 1 in [18]). By adjusting the prism insertion, pulses as short as $26 \mathrm{fs}$ with $\sim 1 \mathrm{~nJ}$ pulse energy were obtained at a repetition rate of $85 \mathrm{MHz}$. We believe these are the shortest pulses reported from SBR modelocked Cr:colquiriite lasers. Figure $\underline{4}$ (top) shows the optical spectrum, centered around $\sim 850 \mathrm{~nm}$, with an FWHM of $\sim 33 \mathrm{~nm}$. The measured pulse width of $\sim 26 \mathrm{fs}$ [Fig. $\underline{4}$ (bottom)] was slightly larger than transform limited ( $23 \mathrm{fs}$ ), with a timebandwidth product of $\sim 0.36$. Figure 4 (top) also shows the calculated reflectivity for the $850 \mathrm{~nm}$ SBR, indicating that the spectral bandwidths (or the pulse widths) are limited by the SBR bandwidth.

\section{FEMTOSECOND TUNING OF CR:LISAF AROUND 800 NM}

\section{A. Experimental Setup}

The experimental setup for the femtosecond tuning experiments with Cr:LiSAF around $800 \mathrm{~nm}$ was similar to that described in Subsection 2.A for $850 \mathrm{~nm}$. The $850 \mathrm{~nm}$ SBR was replaced with a $800 \overline{\mathrm{nm}} \mathrm{SBR}$ and the output coupler 
transmission was $1 \%$. The $800 \mathrm{~nm}$ SBR consisted of 25 pairs of $\mathrm{Al}_{0.95} \mathrm{Ga}_{0.05} \mathrm{As} / \mathrm{Al}_{0.17} \mathrm{Ga}_{0.83} \mathrm{As}$ quarter-wave layers for the Bragg mirror stack and five layers of $6 \mathrm{~nm}$ thick GaAs quantum wells as the saturable absorber. Two pairs of $\mathrm{SiO}_{2}-\mathrm{TiO}_{2}$ layers were used as an HR coating. The calculated modulation depth of the $800 \mathrm{~nm}$ SBR was approximately $0.6 \pm 0.15 \%$.

\section{B. Results}

Figure $\underline{5}$ summarizes the tuning of the femtosecond Cr:LiSAF laser around $800 \mathrm{~nm}$. By rotating the tuning plate, the central wavelength was tuned from 803 to $831 \mathrm{~nm}$. On average, the Cr:LiSAF laser generated $\sim 140 \mathrm{fs}$ pulses with $\sim 90 \mathrm{~mW}$ of average power at a repetition rate of $83 \mathrm{MHz}(\sim 1 \mathrm{~nJ}$ pulse energy). The tuning range was limited by the SBR reflectivity and dispersion bandwidth on the long wavelength side (see Fig. 6). As the laser is tuned above $825 \mathrm{~nm}$, the average output power decreases (Fig. 5) due to the increased SBR loss. Above $825 \mathrm{~nm}$, the reduced output power and the higher cavity dispersion also caused an increase in pulse widths (Fig. 5).

The limitation to tuning below $803 \mathrm{~nm}$ is not clear and may be due to several factors. As Fig. $\underline{6}$ shows, the total cavity dispersion estimate is not flat below $\sim 800 \mathrm{~nm}$. First, the dispersion of the $800 \mathrm{~nm}$ SBR deviated from $\sim 0 \mathrm{fs}^{2}$ below $775 \mathrm{~nm}$ and above $825 \mathrm{~nm}$. Second, the dispersion bandwidth of the custom-designed DCMs was 800-940 nm, and, hence, the dispersion of the cavity was not optimized below $800 \mathrm{~nm}$. By decreasing the pump power, femtosecond tuning in the 791-795 $\mathrm{nm}$ range was also obtained (the wavelength jumped from 795 to $803 \mathrm{~nm}$ ). By using Gires-Tournois interferometer (GTI) mirrors for dispersion compensation and using the same SBR at $800 \mathrm{~nm}$, the laser could be continuously tuned from 794 to $825 \mathrm{~nm}$ (with $~ 300 \mathrm{fs}$ long pulses). This suggests that the jump in tuning is due to the DCM mirrors. Also, cw tuning experiments showed that Cr:LiSAF can lase down to $775 \mathrm{~nm}$. Hence, with a different DCM design, a broader continuous tuning range should be possible around $800 \mathrm{~nm}$.

Finally, using the $800 \mathrm{~nm}$ SBR, pulses as short as $41 \mathrm{fs}$ could be obtained from the Cr:LiSAF laser with a fused-silica prism pair for fine dispersion tuning. This result has been described in an earlier publication (Fig. 6 in [18]).

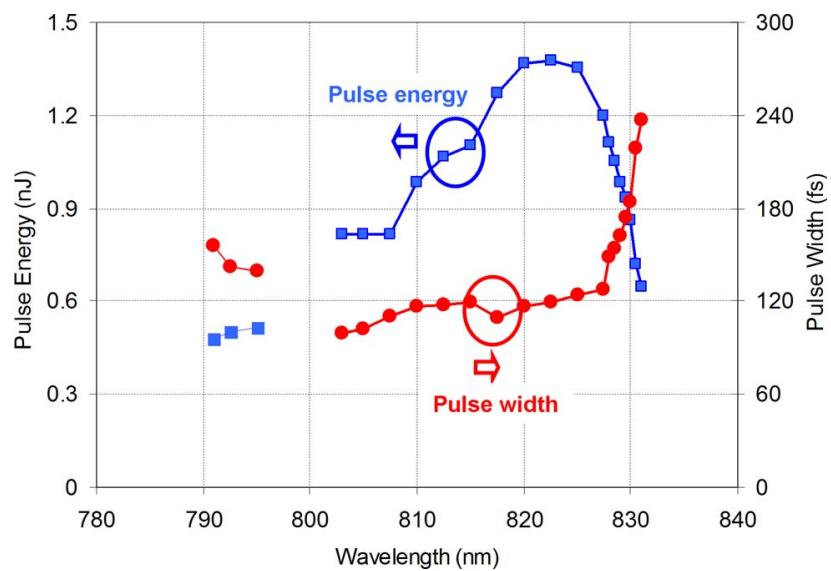

Fig. 5. (Color online) Femtosecond tuning results for the Cr:LiSAF laser with the $800 \mathrm{~nm}$ SBR. The total tuning range is $\sim 28 \mathrm{~nm}$ (803 to $831 \mathrm{~nm})$. At a reduced pump power level, the laser also tuned from 791 to $795 \mathrm{~nm}$.

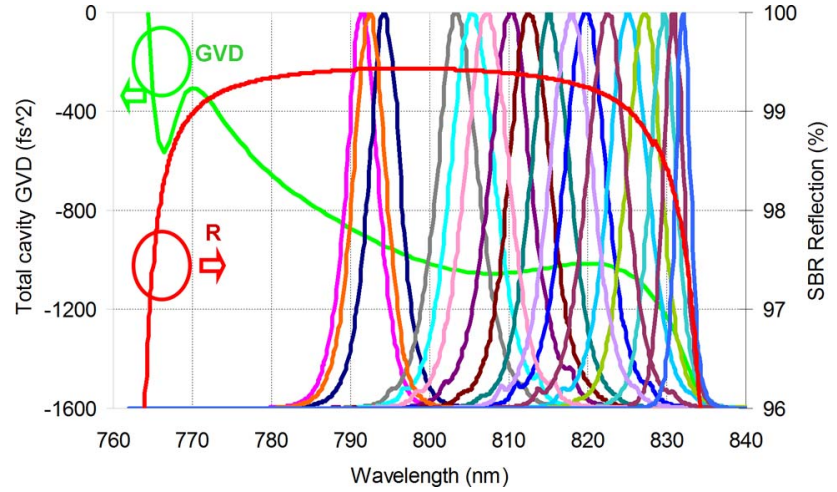

Fig. 6. (Color online) Typical spectra from the Cr:LiSAF laser showing tunability of central wavelength from 803 to $831 \mathrm{~nm}$ for $\sim 140 \mathrm{fs}$ pulses. The data were taken with the $800 \mathrm{~nm}$ SBR. Calculated small-signal reflectivity of the SBR and estimated total cavity GVD are also shown.

\section{FEMTOSECOND TUNING OF THE CR: LISAF LASER AROUND 910 NM}

The experimental setup for the femtosecond tuning experiments with the Cr:LiSAF laser around $900 \mathrm{~nm}$ was also similar to that described for 800 and $850 \mathrm{~nm}$ operation. The $910 \mathrm{~nm}$ SBR consisted of 25 pairs of $\mathrm{Al}_{0.95} \mathrm{Ga}_{0.05} \mathrm{As} / \mathrm{Al}_{0.17} \mathrm{Ga}_{0.83} \mathrm{As}$ quarter-wave layers for the Bragg mirror stack and a $15 \mathrm{~nm}$ thick $\mathrm{In}_{0.10} \mathrm{Ga}_{0.90}$ As quantum well as the saturable absorber. The calculated modulation depth of the $910 \mathrm{~nm}$ SBR was $1.2 \pm 0.3 \%$. With the $910 \mathrm{~nm}$ SBR, broad continuous femtosecond tuning could not be achieved. When the tuning plate was rotated, continuous tuning was generally obtained only over a $10-15 \mathrm{~nm}$ range. Tuning at other wavelengths required adjustment of the focus onto the SBR and cavity dispersion (by changing the number of bounces on DCM mirrors). We believe that this behavior might be caused by dispersion from water absorption lines in air around these wavelengths. Figure 7 summarizes the tuning results around $900 \mathrm{~nm}$, while Fig. $\overline{8}$ shows the output spectra. We stress that, unlike the previous results, the laser was realigned in order to obtain the data shown in Figs. 7 and $\underline{8}$. To cover the full range, not only was the tuning plate rotated, but the SBR position was changed (to adjust the focus on the SBR) and/or the amount of cavity dispersion was changed. The tuning range covers 890 to $922 \mathrm{~nm}$. The average output power was $>100 \mathrm{~mW}$

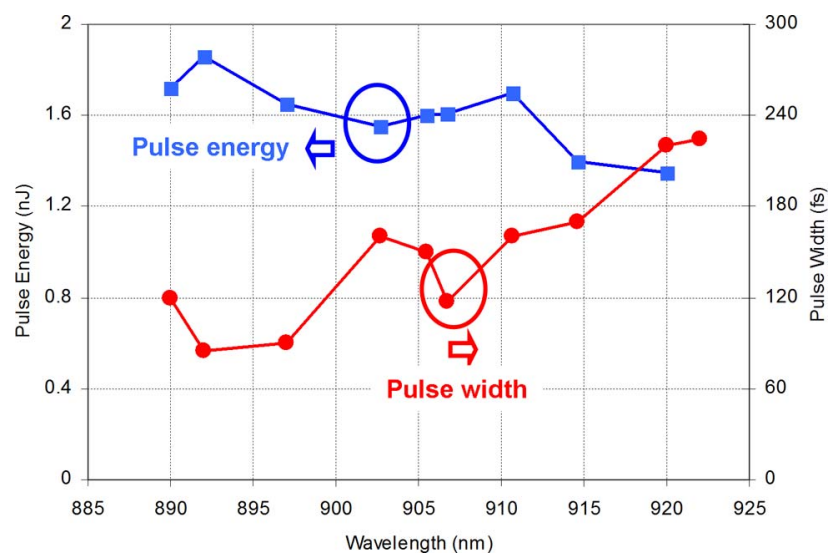

Fig. 7. (Color online) Femtosecond tuning results for the Cr:LiSAF laser using the $910 \mathrm{~nm}$ SBR. The total tuning range is $\sim 32 \mathrm{~nm}$ (890 to $922 \mathrm{~nm}$ ). Obtaining the whole tuning range required adjustment of the focus on the SBR and/or the cavity dispersion level. 




Fig. 8. (Color online) Sample spectra from the Cr:LiSAF laser, showing tunability of central wavelength from 890 to $922 \mathrm{~nm}$. Tuning over the whole range required additional adjustment of the focus on the SBR and/or the cavity dispersion level. The data were taken with the $910 \mathrm{~nm}$ SBR. Calculated small-signal reflectivity of the SBR is also shown.

( $>1.2 \mathrm{~nJ}$ pulse energy) and the pulse width varied between 75 and $300 \mathrm{fs}$. We believe that tuning below $890 \mathrm{~nm}$ is limited by the dispersion and reflectivity bandwidth of the SBR, while tuning above $922 \mathrm{~nm}$ is limited by the absorption edge of the bulk absorber in the SBR (around $\sim 920 \mathrm{~nm}$ ). A broader and continuous femtosecond tuning range around $900 \mathrm{~nm}$ might be achievable using an SBR with a lower modulation depth (and a lower tendency for $Q$-switched mode locking [34]), since the $1.2 \%$ modulation depth of the $910 \mathrm{~nm} \mathrm{SBR}$ is more than required to initiate/sustain cw mode-locked operation for Cr:LiSAF. Moreover, a quantum-well absorber design, rather than the bulk absorber used in this study, and a different band-edge position should improve the femtosecond tuning results.

\section{FEMTOSECOND TUNING OF CR:LICAF AROUND 800 NM}

\section{A. Experimental Setup}

Figure 9 shows the schematic for the Cr:LiCAF laser used for femtosecond tuning around $800 \mathrm{~nm}$. The pump geometry is the same as previously described for the Cr:LiSAF laser. A $2.5 \mathrm{~mm}$ long, 11\% chromium-doped Cr:LiCAF crystal was used as the gain medium; it absorbed $>700 \mathrm{~mW}$ of the incident pump power $(\sim 800 \mathrm{~mW})$. The $800 \mathrm{~nm}$ SBR was used to initiate and sustain mode locking. The output coupler had a transmission of about $2 \%$ around $800 \mathrm{~nm}$. Commercially available pump mirrors, DCM mirrors, and GTI mirrors (Layertec, inc.) were used. In Fig. $\underline{9}$, mirrors M1-M3 are standard pump mirrors with low dispersion over the 750-850 nm wavelength range. Dispersion compensation is accomplished using the GTI and DCM mirrors. High-dispersion GTI mirrors were used because too many bounces on DCM mirrors would be required to cancel dispersion from the Cr:LiCAF crystal (length $\sim 5 \mathrm{~mm}, \mathrm{GVD} \sim 125 \mathrm{fs}^{2}$ ), the quartz birefringent tuning plate (length $\sim 7.3 \mathrm{~mm}, \quad$ GVD $\sim 260 \mathrm{fs}^{2}$ ), and air (length $3.5 \mathrm{~m}, \mathrm{GVD} \sim 70 \mathrm{fs}^{2}$ ). The DCM mirrors had a GVD of only $\sim 45 \pm 10 \mathrm{fs}^{2}$ per bounce $(750-920 \mathrm{~nm})$, whereas the GTI mirrors had $\sim-550 \pm 50 \mathrm{fs}^{2}$ of GVD per bounce. However, the dispersion bandwidth of the GTI mirrors was narrow (790-815 nm), and dispersion oscillated strongly outside this range. In femtosecond tuning experiments, a flat dispersion profile is desired to obtain optimal tuning. Hence, using GTI mirrors limits the tunability range.

\section{B. Results}

Figure 10 summarizes the Cr:LiCAF tuning results, showing the measured pulse widths and pulse energies within the tuning range. The laser was continuously tunable from 785 to $817 \mathrm{~nm}(\sim 32 \mathrm{~nm})$ by rotating the birefringent tuning plate. Figure 11 shows the output spectra along with the estimated total cavity dispersion and SBR reflectivity bandwidth. Tuning was limited by the dispersion bandwidth of the GTI mirror on the short wavelength side. The average pulse duration was $133 \mathrm{fs}$ and the average pulse energy was $1.48 \mathrm{~nJ}$. The laser repetition rate was $86 \mathrm{MHz}$. The $800 \mathrm{~nm}$ SBR reflectivity spectra in Fig. 11 is shifted to shorter wavelengths compared to Fig. $\underline{6}$, which is a different SBR sample where the reflectivity shift is a result of variations in growth conditions (same SBR design but different MBE growth). Except for this small reflectivity shift, there was no significant variation in the SBR parameters. In the tuning studies with Cr:LiCAF, the $800 \mathrm{~nm}$ SBR with a reflectivity shifted toward shorter wavelengths was used, since obtaining shorter wavelength tuning is important for applications such as multiphoton microscopy or amplifier seeding.

To confirm that the GTI mirror dispersion bandwidth limited tuning below $785 \mathrm{~nm}$, the cavity dispersion was made more negative by increasing the number of GTI mirror bounces. Using two bounces on the GTI mirror, tuning in the $775-785 \mathrm{~nm}$ region (12-150 fs, $1.6 \mathrm{~nJ}$ pulses), and with three bounces on the GTI mirror, tuning in the $765-775 \mathrm{~nm}$ region ( $170 \mathrm{fs}, 1.37 \mathrm{~nJ}$ pulse at $767 \mathrm{~nm}$ ) could be obtained. Figure 12 shows the mode-locked spectra together with the reflectivity of the $800 \mathrm{~nm}$ SBR. Tuning above $817 \mathrm{~nm}$ is limited by the SBR bandwidth, which was also apparent from a decrease in output power (Fig. 9). The total tuning range extends

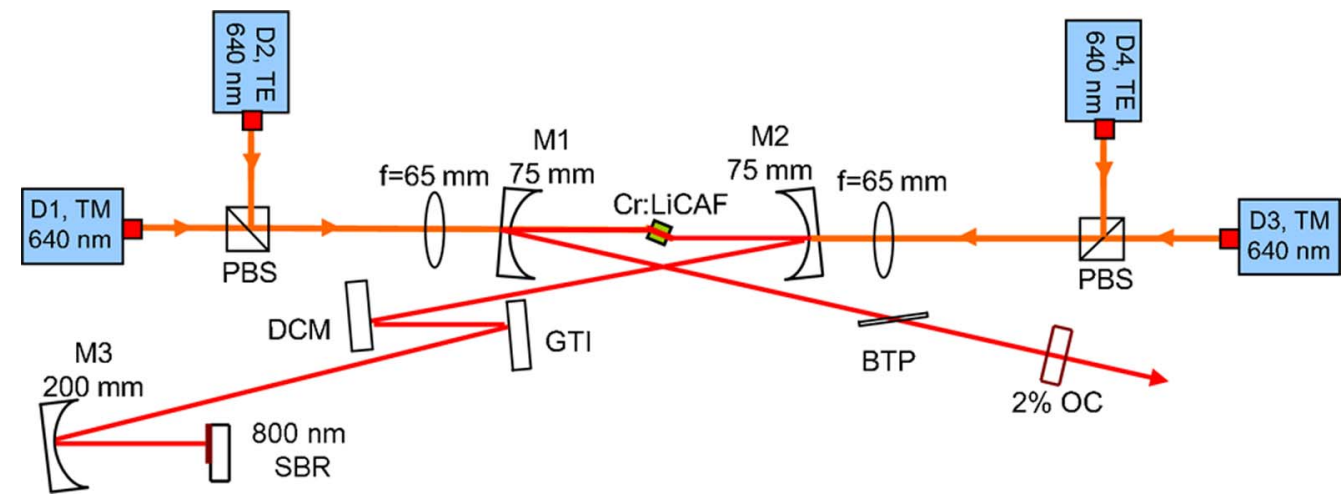

Fig. 9. (Color online) Schematic of the single-mode diode-pumped Cr:LiCAF laser used in femtosecond tuning experiments around 800 nm. 


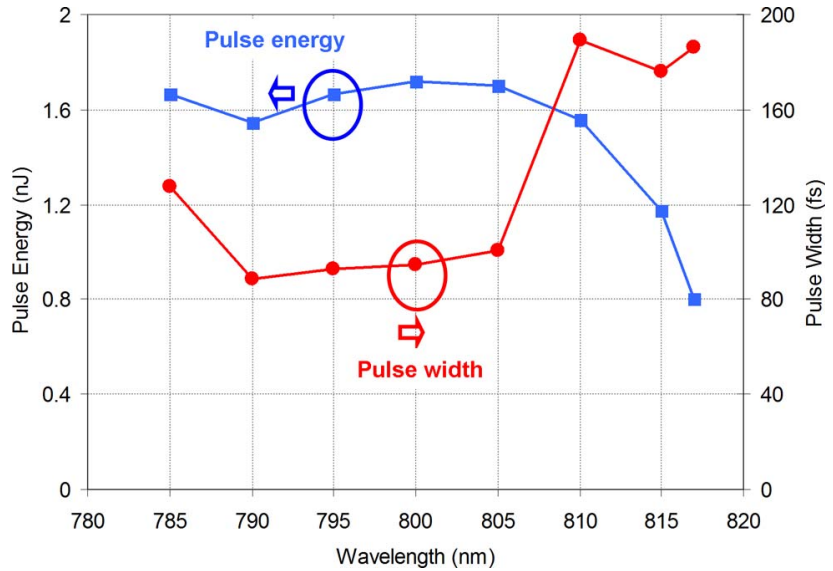

Fig. 10. (Color online) Femtosecond tuning results for the Cr:LiCAF laser using the $800 \mathrm{~nm}$ SBR. The laser was continuously tunable from 785 to $817 \mathrm{~nm}$ (32 $\mathrm{nm}$ bandwidth).

from 767 to $817 \mathrm{~nm}(50 \mathrm{~nm})$, but multiple dispersion settings were required to cover this wavelength range. However, this suggests that, with a different DCM mirror having flat dispersion, it should be possible to obtain continuous tuning.

Last, we have generated ultrashort pulses with a Cr:LiCAF laser using DCM mirrors. By using four bounces (eight per round trip) on the DCM mirrors, a total cavity GVD of about $-100 \mathrm{fs}^{2}$ around $800 \mathrm{~nm}$ could be obtained in one round trip. In this configuration, the laser produced $\sim 39 \mathrm{fs}$ pulses with $125 \mathrm{~mW}$ of average output power at a repetition rate of $77 \mathrm{MHz}$, with an absorbed pump power of $\sim 700 \mathrm{~mW}$. The corresponding pulse energy was 1.62 nJ. Figure 13 (top) shows the optical spectrum as well as the estimated cavity dispersion. Figure 13 (bottom) shows the measured pulse autocorrelation. The optical spectrum was centered around $\sim 805 \mathrm{~nm}$ and had a bandwidth of $18.8 \mathrm{~nm}$, broad enough to support $\sim 36 \mathrm{fs}$ pulses. The dispersion of the cavity was around $-100 \mathrm{fs}^{2}$ at the center of the optical spectrum, and deviates at the extremes due to the relatively narrow dispersion bandwidth of the SBR. Figure 13 shows that the optical spectrum covers a significant portion of the SBR bandwidth. Based on results from the Cr:LiSAF laser, the SBR should have enough bandwidth to obtain pulses as short as $-25 \mathrm{fs}^{2}$. Hence, we have

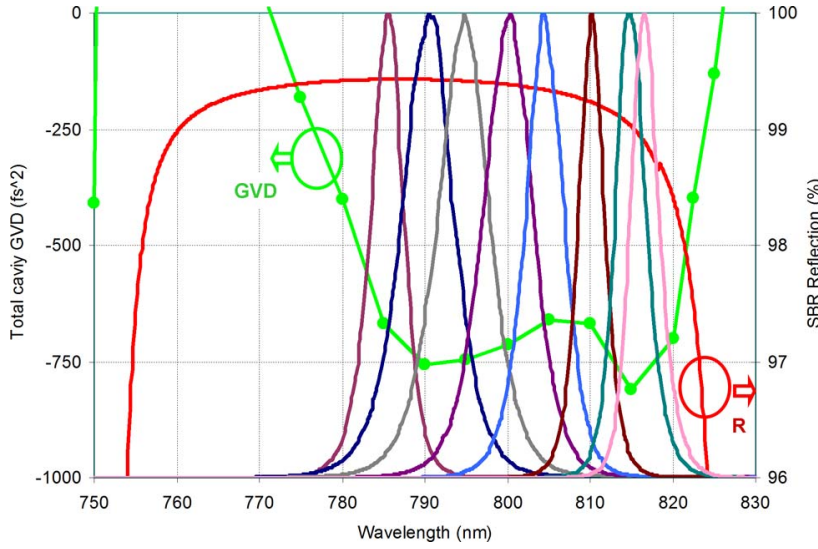

Fig. 11. (Color online) Typical spectra from the mode-locked Cr:LiCAF laser, showing tunability of the center wavelength from 785 to $817 \mathrm{~nm}$. The data were taken with the $800 \mathrm{~nm}$ SBR. Calculated small-signal reflectivity of the SBR and estimated total cavity GVD are also shown.

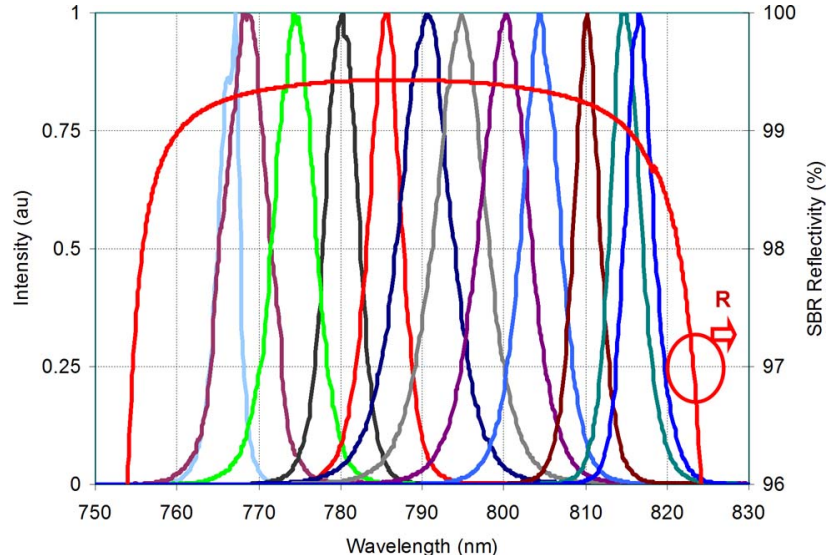

Fig. 12. (Color online) Full tuning range obtained with the Cr:LiCAF laser using the $800 \mathrm{~nm}$ SBR. The calculated reflectivity of the SBR is also shown. The total tuning range extends from 767 to $817 \mathrm{~nm}(50 \mathrm{~nm}$ bandwidth).

also tried to obtain even shorter pulses with a Cr:LiCAF laser from a cavity including a prism pair for fine dispersion tuning. However, $Q$-switched mode-locking, multiple-pulsing, and cwbreakthrough instabilities prevented us from obtaining stable cw-mode-locked operation with shorter pulses. We believe that this is due to the lower emission cross section of the Cr:LiCAF gain media [19], which results in a very narrow SBR mode-locking ranges for sub-50-fs pulses [35].
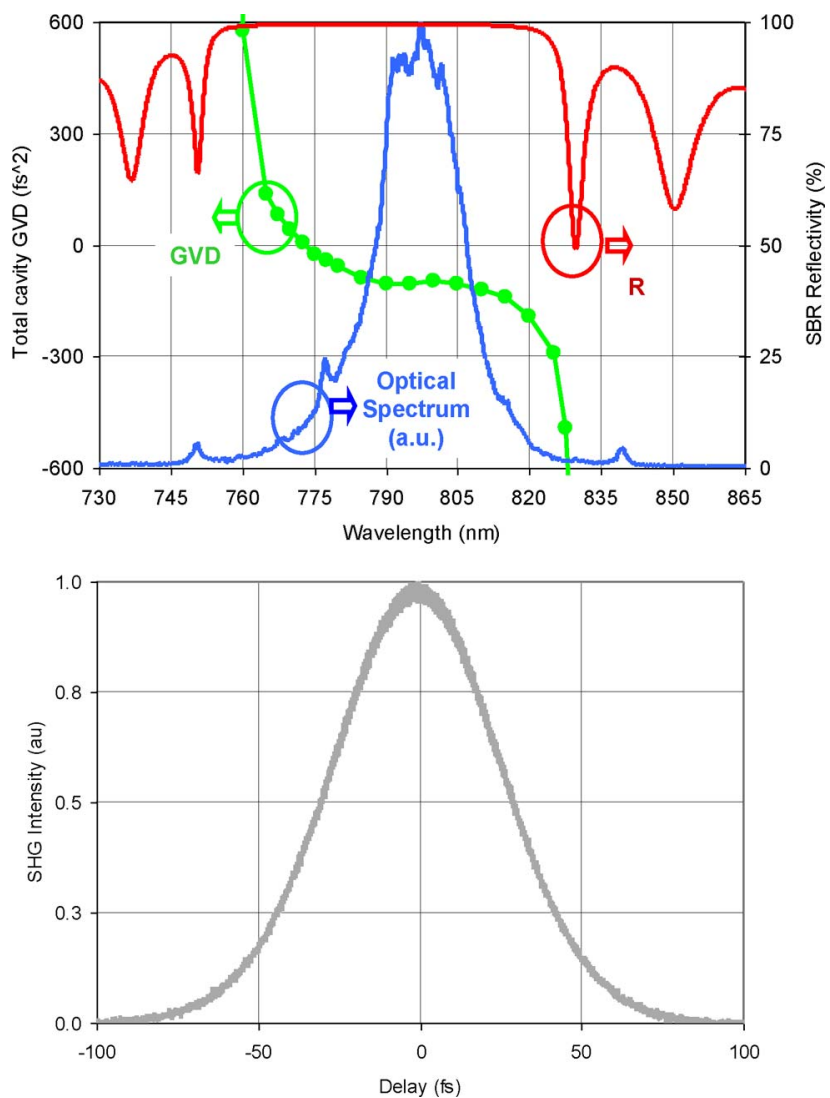

Fig. 13. (Color online) Top, optical spectrum of the $\sim 39 \mathrm{fs}, 1.62 \mathrm{~nJ}$ pulses and the calculated reflectivity curve for the $800 \mathrm{~nm}$ SBR and estimated total cavity dispersion. Bottom, measured background-free autocorrelation trace shows pulse durations of $39 \mathrm{fs}$. 


\section{SUMMARY AND DISCUSSION}

This manuscript presents a comprehensive study of femtosecond tuning of Cr:colquiriite lasers pumped by single-mode diodes. In each cavity configuration, two different regimes of tunable mode-locked operation were demonstrated: (1) broad, continuous femtosecond tuning at higher negative intracavity dispersion and (2) sub-50-fs pulse generation spanning the spectral bandwidth of the SBR at intracavity dispersions closer to zero. A quartz birefringent plate was used for tuning, which was easier and more robust compared to tuning with a prism pair and a slit. With the Cr:LiSAF gain media, and an $850 \mathrm{~nm}$ SBR, a femtosecond tuning range of $828-873 \mathrm{~nm}$ (45 nm) was obtained with average pulse widths of $\sim 190 \mathrm{fs}$ and average pulse energies of $1.87 \mathrm{~nJ}$. In addition, a femtosecond tuning range of $835-870 \mathrm{~nm}(35 \mathrm{~nm})$ was obtained with average pulse widths of $\sim 140 \mathrm{fs}$ and average pulse energies of $1.8 \mathrm{~nJ}$. In both cases, tuning was performed by rotating the birefringent plate. With the $800 \mathrm{~nm}$ SBR, femtosecond tuning of the Cr:LiSAF laser was obtained in the $803-831 \mathrm{~nm}$ range (28 nm) with average pulse widths of $\sim 140 \mathrm{fs}$ and average pulse energies of $1 \mathrm{~nJ}$. We also demonstrated femtosecond tuning of a Cr:LiSAF laser in the $890-923 \mathrm{~nm}$ region, using a $910 \mathrm{~nm}$ SBR. However, both the cavity dispersion and the spot size on the SBR required adjustment to obtain this tuning range. With Cr:LiSAF, the shortest pulse widths obtained using the 800,850 and $910 \mathrm{~nm}$ centered SBRs were 41, 26, and $75 \mathrm{fs}$, respectively.

Using a Cr:LiCAF gain medium, a mode-locked tuning range of $767-817 \mathrm{~nm}(50 \mathrm{~nm})$, with average pulse widths of $\sim 133 \mathrm{fs}$ and average pulse energies of $1.48 \mathrm{~nJ}$ was obtained. The laser could be tuned by rotating the birefringent plate in the $785-817 \mathrm{~nm}$ region, but required dispersion adjustment to tune below $785 \mathrm{~nm}$. We believe that, with a different DCM mirror design, the entire tuning range can be continuously covered. Pulses as short as $~ 39$ fs were obtained, which we believe are the shortest pulses from SBR mode-locked Cr:LiCAF lasers.

The total femtosecond tuning range that was demonstrated in this study extends from 767 to $923 \mathrm{~nm}$. The total $\mathrm{cw}$ tuning range that was demonstrated by Cr:colquiriites covers the 754-1042 nm region. We envision that it should be possible to cover this full $\mathrm{cw}$ tuning range with improved SBRs and DCMs.

Because of the limited reflectivity and dispersion bandwidth, the broadest obtainable tuning range using a single SBR is about $50 \mathrm{~nm}$ using AlGaAs-based SBRs. The bandwidth of the SBR also limited the pulse widths; however, $\sim 26 \mathrm{fs}$ pulses were still obtained. In the future, the use of oxidized broadband SBR mirrors [36] may enable the generation of pulses as short as $10 \mathrm{fs}[13,22]$, or broadly tunable sub-100-fs pulses from $\sim 800$ to $\sim 1000 \mathrm{~nm}$. Also, the peak power that was obtained in this study was in the $20-40 \mathrm{~kW}$ range and was limited by the available pump power $(\sim 800 \mathrm{~mW})$. However, earlier studies have shown that the peak power can be scaled up to the $\sim 100 \mathrm{~kW}$ level by multipass cavity Cr:colquiriite lasers at an $\sim 10 \mathrm{MHz}$ repetition rate [37], or to the $\sim 1 \mathrm{MW}$ level by cavity-dumped Cr:colquiriite lasers with pulse repetition rates of up to $50 \mathrm{kHz}$ [38]. Moreover, improvements in pump diode output power are expected to enable peak power scaling. For example, recently, a $1 \mathrm{~W}$, nearly transform-limited $\left(M^{2} \sim 1.3\right)$ $650 \mathrm{~nm}$ diode laser has been demonstrated (which is approximately 5 times brighter than the pump laser diodes that were used in this study) [39]. We believe that tunable femtosecond Cr:colquiriite lasers have the potential to become a versatile, low-cost femtosecond source for several important application areas in science and engineering, including multiphoton microscopy $[\underline{1,40]}$.

\section{ACKNOWLEDGMENTS}

We thank P. Fendel, J. Wang, J. Birge, Y. Hu, S. Nabanja, and H. Byun for experimental help and acknowledge partial support by U.S. Air Force Office of Scientific Research (USAFOSR) contracts FA9550-07-1-0014 and FA9550-07-1-0101, National Science Foundation (NSF) ECCS-0900901, and Thorlabs Inc.

\section{REFERENCES}

1. C. Xu, W. Zipfel, J. B. Shear, R. M. Williams, and W. W. Webb, "Multiphoton fluorescence excitation: New spectral windows for biological nonlinear microscopy," Proc. Natl. Acad. Sci. USA 93, 10763-10768 (1996).

2. S. A. Payne, L. L. Chase, L. K. Smith, W. L. Kway, and H. W. Newkirk, "Laser performance of $\mathrm{LiSAIF}_{6}: \mathrm{Cr}^{3+}$, "J. Appl. Phys. 66, 1051-1056 (1989).

3. L. K. Smith, S. A. Payne, W. L. Kway, L. L. Chase, and B. H. T. Chai, "Investigation of the laser properties of $\mathrm{Cr}^{3+}: \mathrm{LiSrGaF}_{6}$," IEEE J. Quantum Electron. 28, 2612-2618 (1992).

4. S. A. Payne, L. L. Chase, H. W. Newkirk, L. K. Smith, and W. F. Krupke, "LiCaAlF 6 : $\mathrm{Cr}^{3+}$ a promising new solid-state laser material,” IEEE J. Quantum Electron. 24, 2243-2252 (1988).

5. R. Scheps, J. F. Myers, H. B. Serreze, A. Rosenberg, R. C. Morris, and M. Long, "Diode-pumped Cr:LiSrAlF 6 laser," Opt. Lett. 16, 820-822 (1991).

6. P. M. W. French, R. Mellish, J. R. Taylor, P. J. Delfyett, and L. T. Florez, "Mode-locked all-solid-state diode-pumped Cr:LiSAF laser," Opt. Lett. 18, 1934-1936 (1993).

7. S. Tsuda, W. H. Knox, and S. T. Cundiff, "High efficiency diode pumping of a saturable Bragg reflector-mode-locked Cr:LiSAF femtosecond laser," Appl. Phys. Lett. 69, 1538-1540 (1996).

8. D. Kopf, K. J. Weingarten, G. Zhang, M. Moser, M. A. Emanuel, R. J. Beach, J. A. Skidmore, and U. Keller, "High-average-power diode-pumped femtosecond Cr:LiSAF lasers,” Appl. Phys. B 65 235-243 (1997).

9. G. J. Valentine, J. M. Hopkins, P. Loza-Alvarez, G. T. Kennedy, W. Sibbett, D. Burns, and A. Valster, "Ultralow-pump-threshold, femtosecond $\mathrm{Cr}^{3+}: \mathrm{LiSrAlF}_{6}$ laser pumped by a single narrowstripe AlGaInP laser diode," Opt. Lett. 22, 1639-1641 (1997).

10. J. M. Hopkins, G. J. Valentine, W. Sibbett, J. A. der Au, F. MorierGenoud, U. Keller, and A. Valster, "Efficient, low-noise, SESAMbased femtosecond $\mathrm{Cr}^{3+}{ }^{\mathrm{LiShAlF}} 6$ laser," Opt. Commun. 154, 54-58 (1998).

11. B. Agate, B. Stormont, A. J. Kemp, C. T. A. Brown, U. Keller, and W. Sibbett, "Simplified cavity designs for efficient and compact femtosecond Cr:LiSAF lasers," Opt. Commun. 205, 207-213 (2002).

12. J. M. Hopkins, G. J. Valentine, B. Agate, A. J. Kemp, U. Keller, and W. Sibbett, "Highly compact and efficient femtosecond $\mathrm{Cr}$ : LiSAF lasers," IEEE J. Quantum Electron. 38, 360-368 (2002).

13. P. Wagenblast, R. Ell, U. Morgner, F. Grawert, and F. X. Kärtner, "Diode-pumped $10 \mathrm{fs} \mathrm{Cr}^{3+}$ :LiCAF laser," Opt. Lett. 28, 1713-1715 (2003).

14. A. Isemann and C. Fallnich, "High-power colquiriite laser with high slope efficiencies pumped by broad-area laser diodes," Opt. Express 11, 259-264 (2003).

15. R.P. Prasankumar, Y.Hirakawa, A. M. J. Kowalevicz, F. X. Kärtner, J. G. Fujitimo, and W. H. Knox, "An extended cavity femtosecond Cr:LiSAFlaser pumped by low cost diode lasers," Opt. Express 11, 1265-1269 (2003).

16. U. Demirbas, A. Sennaroglu, A. Benedick, A. Siddiqui, F. X. Kärtner, and J. G. Fujimoto, "Diode-pumped, high-average power femtosecond $\mathrm{Cr}^{+3}$ :LiCAF laser," Opt. Lett. 32, 3309-3311 (2007). 
17. U. Demirbas, A. Sennaroglu, F. X. Kärtner, and J. G. Fujimoto, "Highly efficient, low-cost femtosecond $\mathrm{Cr}^{3+}: \mathrm{LiCAF}$ laser pumped by single-mode diodes," Opt. Lett. 33, 590-592 (2008).

18. U. Demirbas, D. Li, J. R. Birge, A. Sennaroglu, G. S. Petrich, L. A. Kolodziejski, F. X. Kärtner, and J. G. Fujimoto, "Low-cost, singlemode diode-pumped Cr:colquiriite lasers," Opt. Express 17, 14374-14388 (2009).

19. U. Demirbas, A. Sennaroglu, F. X. Kärtner, and J. G. Fujimoto, "Comparative investigation of diode pumping for continuouswave and mode-locked $\mathrm{Cr}^{3+}$ :LiCAF lasers," J. Opt. Soc. Am. B 26, 64-79 (2009).

20. H. Maestre, A. J. Torregrosa, J. A. Pereda, C. R. FernandezPousa, and J. Capmany, "Dual-wavelength $\mathrm{Cr}^{3+}: \mathrm{LiCaAlF}_{6}$ solid-state laser with tunable $\mathrm{THz}$ frequency difference," IEEE J. Quantum Electron. 46, 1681-1685 (2010).

21. J. F. Pinto, L. Esterowitz, and G. H. Rosenblatt, "Frequency tripling of a Q-switched Cr:LiSAF laser to the UV region," IEEE J. Sel. Top. Quantum Electron. 1, 58-61 (1995).

22. S. Uemura and K. Torizuka, "Generation of $10 \mathrm{fs}$ pulses from a diode-pumped Kerr-lens mode-locked Cr:LiSAF laser,” Jpn. J. Appl. Phys. 39, 3472-3473 (2000).

23. I. T. Sorokina, E. Sorokin, E. Wintner, A. Cassanho, H. P. Jenssen, and R. Szipocs, "14 fs pulse generation in Kerr-lens mode-locked prismless Cr:LiSGaF and Cr:LiSAF lasers: observation of pulse self-frequency shift," Opt. Lett. 22, 1716-1718 (1997).

24. P. Wagenblast, U. Morgner, F. Grawert, V. Scheuer, G. Angelow, M. J. Lederer, and F. X. Kärtner, "Generation of sub-10-fs pulses from a Kerr-lens mode-locked $\mathrm{Cr}^{3+}: \mathrm{LiCAF}$ laser oscillator using third-order dispersion-compensating double-chirped mirrors," Opt. Lett. 27, 1726-1729 (2002).

25. R. Mellish, S. C. W. Hyde, N. P. Barry, R. Jones, P. M. W. French, J. R. Taylor, C. J. van der Poel, and A. Valster, "All-solid-state diode-pumped Cr:LiSAF femtosecond oscillator and regenerative amplifier," Appl. Phys. B 65, 221-226 (1997).

26. A. Robertson, R. Knappe, and R. Wallenstein, "Diode-pumped broadly tunable (809-910 nm) femtosecond Cr:LiSAF laser," Opt. Commun. 147, 294-298 (1998).

27. E. Sorokin, "Solid-state materials for few-cycle pulse generation and amplification," in Few-Cycle Laser Pulse Generation and Its Applications, F. X. Kärtner, ed. (Springer-Verlag, 2004), pp. $3-71$.

28. S. Tsuda, W. H. Knox, S. T. Cundiff, W. Y. Jan, and J. E. Cunningham, "Mode-locking ultrafast solid-state lasers with saturable Bragg reflectors," IEEE J. Sel. Top. Quantum Electron. 2, 454-464 (1996).
29. U. Keller, K. J. Weingarten, F. X. Kärtner, D. Kopf, B. Braun, I. D Jung, R. Fluck, C. Hönninger, N. Matuschek, and J. A. der Au, "Semiconductor saturable absorber mirrors (SESAM's) for femtosecond to nanosecond pulse generation in solid-state lasers," IEEE J. Sel. Top. Quantum Electron. 2, 435-453 (1996).

30. D. Kopf, A. Prasad, G. Zhang, M. Moser, and U. Keller, "Broadly tunable femtosecond Cr:LiSAF laser," Opt. Lett. 22, 621-623 (1997).

31. U. Demirbas, "Low-cost, highly efficient and tunable ultrafast laser technology based on directly diode pumped Cr:colquiriites," Ph.D. dissertation (Massachusetts Institute of Technology, Department of Electrical Engineering and Computer Science, 2010).

32. J. R. Birge and F. X. Kärtner, "Efficient optimization of multilayer coatings for ultrafast optics using analytic gradients of dispersion,” Appl. Opt. 46, 2656-2662 (2007).

33. D. Li, U. Demirbas, J. R. Birge, G. S. Petrich, L. A. Kolodziejski, A. Sennaroglu, F. X. Kärtner, and J. G. Fujimoto, "Diode-pumped passively mode-locked $\mathrm{GHz}$ femtosecond Cr:LiSAF laser with kW peak power," Opt. Lett. 35, 1446-1448 (2010).

34. C. Hönninger, R. Paschotta, F. Morier-Genoud, M. Moser, and U. Keller, " $Q$-switching stability limits of continuous-wave passive mode locking," J. Opt. Soc. Am. B 16, 46-56 (1999).

35. F. X. Kärtner, L. R. Brovelli, D. Kopf, M. Kamp, I. Calasso, and U. Keller, "Control of solid-state laser dynamics by semiconductor devices," Opt. Eng. 34, 2024-2036 (1995).

36. S. N. Tandon, J. T. Gopinath, H. M. Shen, G. S. Petrich, L. A. Kolodziejski, F. X. Kärtner, and E. P. Ippen, "Large-area broadband saturable Bragg reflectors by use of oxidized AlAs," Opt. Lett. 29, 2551-2553 (2004).

37. U. Demirbas, A. Sennaroglu, F. X. Kärtner, and J. G. Fujimoto, "Generation of $15 \mathrm{~nJ}$ pulses from a highly efficient, low-cost multipass-cavity $\mathrm{Cr}^{3+}$ :LiCAF laser," Opt. Lett. 34, 497-499 (2009)

38. U. Demirbas, K.-H. Hong, J. G. Fujimoto, A. Sennaroglu, and F. X. Kärtner, "A low-cost cavity-dumped femtosecond $\mathrm{Cr}^{3+}$ :LiSAF laser producing > 100 nJ pulses," Opt. Lett. 35 (2010).

39. P. Adamiec, B. Sumpf, I. Rudiger, J. Fricke, K. H. Hasler, P Ressel, H. Wenzel, M. Zorn, G. Erbert, and G. Trankle, "Tapered lasers emitting at $650 \mathrm{~nm}$ with $1 \mathrm{~W}$ output power with nearly diffraction-limited beam quality," Opt. Lett. 34, 2456-2458 (2009).

40. S. Sakadžić, U. Demirbas, T. R. Mempel, A. Moore, S. Ruvinskaya, D. A. Boas, A. Sennaroglu, F. X. Kärtner, and J. G. Fujimoto, "Multi-photon microscopy with a low-cost and highly efficient Cr:LiCAF laser," Opt. Express 16, 2084820863 (2008). 